\title{
DE ROMA A MANILA: EL CAMBIO DE LA DEMOGRAFIA EN EL TRANSCURSO DE TRES DÉCADAS*
}

\author{
RONALD LEE* \\ Universidad de California, Berckeley
}

\section{INTRODUCCIÓN}

DURANTE LAS TRES ÚltimaS décadas desde Roma, ${ }^{3}$ la demografía ha formado nuevas alianzas con diversas ciencias sociales; la mayoria de los problemas de cómputo se han vuelto insignificantes ya que gracias a la computadora se han recolectado en el mundo conjuntos de datos a nivel micro tanto sobre actitudes como de tipo descriptivo; hemos aprendido mucho más sobre el mundo, al menos en su aspecto descriptivo; se han multiplicado y vuelto más complejas nuestras teorías; y por doquier el análisis se ha hecho más formal. Los objetivos de este trabajo son tratar de esclarecer estos cambios, y más importante aún, imponer un orden en éstos y describirlos buscando patrones, tendencias y explicaciones.

La demografía no solamente es el estudio cuantitativo de la población y sus cambios, sino también el estudio de las causas y consecuencias de los cambios poblacionales vistas desde una perspectiva más amplia. Por otro lado, como aquí me limito al estudio de estos temas, sólo comentaré brevemente los esfuerzos realizados para influir sobre las tendencias demográficas.

Asimismo, en têrminos generales se excluye de la discusión el importante tema de la divulgación de programas estatales de planificación familiar durante los últimos 20 años, y la evidencia creciente de su eficacia.

Esta discusión consta de cinco partes. Primero serán consideradas las fuentes del cambio en demografía, a saber: la ucumulación de conocimiento, la influencia de la computadora, el surgimiento de nuevas líneas de investigación dentro de la disciplinà misma así como los aportes innovadores de otras disciplinas. Segundo, serán caracterizados los tipos de cambio según otras dimensiones: micro versus macro, descriptivo versus conductista, causas versus consecuencias. Tercero, se dará una visión sintética del campo revisando el status de las teorías de la transición

\footnotetext{
* Trabajo preparado para la reunión de la Unión Internacional para el Estudio Científico de la Población (UIECP) en Manila, Filipinas, diciembre de 1981. El autor agradece a John Bongaarts, Ken Wachter, Paul Demeny, Allan Sharlin y Carl Mosk sus valiosos comentarios, y a los últimos tres junto con Brian Arthur, Al Hermalin y John Hobcraft haber comentado una versión anterior. Fondos del NICHD, en forma de becas números HD4146 y HD14656, apoyaron parte del trabajo.

1 Lugar donde se llevó a cabo el l Congreso Mundial sobre Población, en 1954.
} 
demográfica y la maltusiana. Cuarto, se harán algunas reflexiones sobre cuáles de estas lineas de investigación se aproximan al agotamiento de sus agendas, y cuáles tienen la probabilidad de ser las más dinámicas en la próxima década. Quinto, se ofrecerán algunas conclusiones generales extraídas de esta discusión.

\section{FUENTES DEL CAMBIO EN DEMOGRAFÍA}

La descripción de los cambios puede ordenarse de múltiples maneras. Prefiero verlos como si fueran, el desarrollo económico de algún país: o sea, en términos de un modelo simple del crecimiento y de sus alteraciones sectoriales. Con este propósito, es útil distinguir cuatro fuentes de progreso y cambio estructural dentro de la demografía. Primero -y básico- es el proceso simple de acumulación de conocimiento: se aprende más levantando más censos en más países, replicando análisis similares sobre diversos conjuntos de datos, logrando crecientes mejoras metodológicas, facilitando los esfuerzos de organización y empresa que reúnen equipos de investigadores para enfrentar problemas específicos, etc.

Segundo, el cambio tecnológico fuera de la demografía, y de hecho fuera de las ciencias sociales, ha dado como resultado la computadora. La computadnra no sólo ha hecho más fácil el cálculo, sino también ha privilegiado, de manera diferencial, ciertos tipos de investigación demográfica alterando así la estructura del campo, es decir, la intensidad y la calidad del esfuerzo en varias subáreas.

Tercero, la innovación dentro del campo, la apertura de nuevas líneas de investigación derivadas de una nueva teoría, una nueva metodología o una nueva inquietud. Los modelos biométricos de fecundidad, aplicados a los análisis de las variables intermedias de fecundidad, son un ejemplo de una nueva línea de investigación muy exitosa.

Cuarto, innovaciones importantes han sido introducidas desde fuera de la demografía: así tenemos el caso ejemplar de la teoría económica de la fecundidad.

$\mathrm{Al}$ atribuirse el cambio en demografía a estas cuatro fuentes, se está planteando una visión muy académica de su desarrollo, como si esto ocurriera dentro de un vacío, según leyes intelectuales. Se espera, por supuesto, que la demografía como campo práctico, también responda a tendencias y patrones demográficos reales a nivel mundial. La mortalidad baja de manera más o menos "satisfactoria", la fecundidad sigue demasiado "alta" o se convierte en demasiado "baja"; así es que se estudia la fecundidad en forma más intensa que la mortalidad.

La demografía como campo también se ve afectada por los actores en la formulación de políticas de población y por las agencias donantes que deciden qué áreas son importantes o estimulantes, $y$ en consecuencia otorgan su apoyo a la investigación. En este trabajo se presta escasa atención a estas influencias, en parte, quizá, por ingenuidad, y en parte porque no convence el argumento de que hayan tenido un papel tan decisivo como otras influencias ya descritas. Existen áreas importantísimas para las políticas de población dentro de las cuales poco progreso se ha logrado, y donde el dinero no ha llegado a comprar una investigación exitosa; un ejemplo es el estudio de las consecuencias del cambio poblacional. En cambio, otras áreas han crecido rápidamente sin relevancia inmediata para las políticas de población, como es el caso de la demografía histórica. 


\section{La acumulación sistemática de conocimiento}

La mayor parte del progreso dentro de la demografía es el resultado de una acumulación continua de conocimiento, a través de las contribuciones de demógrafos individuales, de departamentos de estadística del gobiemo, $\mathrm{y}$ de equipos de investigación organizados. No se justifica tratar de cubrir todas estas áreas aquí; sólo se hará mención de algunos ejemplos.

Primero, se han incrementado y mejorado enormemente los datos demográficos básicos para todos los países del mundo. Se ha incrementado drásticamente la proporción de población mundial cubierta por censos comprensivos regulares, y esta proporción dará un salto de gran magnitud con el censo chino del próximo verano. Durante el período que estudiamos, Whelpton, Freedman, Rydier y Westoff desarrollaron la Encuesta Nacional de Fecundidad en los Estados Unidos, la cual fue extendida al contexto de los países menos desarrollados por la encuesta de Chandrasekaran, en Mysore, y por las múltiples encuestas tipo CAP aplicadas en todas las regiones del mundo en vías de desarrollo. Estos esfuerzos han culminado en la Encuesta Mundial de Fecundidad, la cual ha proporcionado una masa de información comparable sobre niveles y tendencias de fecundidad, tamaño de familia deseado, variables intermedias, $y$, en menor grado, aspectos socioeconómicos correlacionados.

La demografía matemática sigue explotando de manera comprensiva y con imaginación el modelo básico de Lotka sobre poblaciones estables, con incursiones en el mundo de poblaciones sistemáticamente no estables; aquí se requiere mencionar a Keyfitz, Bourgeois-Pichat, López, Tabbarah, Coale y Lebras. No obstante su sencillez fundamental, el modelo sigue logrando nuevos alcances y aplicaciones en áreas tan diversas como el momentum poblacional, el status dentro de las jerarquías, el crecimiento económico, las estimaciones indirectas, la frecuencia de parentesco, y los costos de programas para pensionados. Cuánto tiempo más seguirá dando frutos este modelo es tema aparte; la indagación más profunda de estos problemas requiere modelos diferentes.

El análisis de la migración proporciona un buen ejemplo de un creciente desarrollo fructífero, sin grandes sal tos ni grandes sorpresas. Las influencias básicas son bien conocidas y fáciles de replicar en diversos estudios: distançia, masa de población, edad, educación, diferencias en niveles de empleo y de ingreso. Quizá no haya dentro de la demografía otras relaciones con un contenido de comportamiento tan seguro. Muchas de las observaciones básicas están contenidas en las leyes de Ravenstein del siglo pasado. Por supuesto, en este campo la teoría no se ha de tenido, pero los cambios no han sido dramáticos. La aplicación de Sjaastad del enfoque de capital humano a la migración no ha ocasionado grandes conmociones, como lo ha sido su aplicación a la fecundidad. Un novedoso y útil enfoque considera a la familia o el hogar (en vez del individuo) como unidad de decisiones, siendo Mincer y DaVanzo los autores pioneros; otro enfoque valioso es la preocupación por la búsqueda del trabajo y los mercados de trabajo geográficos, articulado por David y Schwartz. El modelo de Todaro, aplicado a los países menos desarrollados, representa una pequeña variación sobre el tema básico, pero tiene implicaciones importantes para las políticas de población y ha contribuido a orientar numerosas investigaciones. El crecimiento a corto plazo de la 
migración internacional obrera en el mundo entero ha motivado la investigación de Tapiños, de varios estudiosos de la OIT, y de muchos otros. En resumen, el estudio de la migración es un campo que ha producido hallazgos empíricos sólidos, donde la teoría aparentemente ha crecido, aumentando y refinando de manera constructiva los enfoques anteriores, en lugar de construir estructuras competitivas paralelas.

\section{La influencia de la computadora}

La segunda fuente de cambios en demografía es tecnológica: la computadora ha hecho posible el almacenamiento y la recuperación de conjuntos de datos grandes y complejos; ha convertido en rutinario el análisis estadistico refinado; y ha permitido diversos tipos de simulación y cálculo. Obviamente estos aspectos de la computadora complementan muchos de los procedimientos y enfoques específicos que han crecido rápidamente dentro de la demografía. Aunque el uso de las encuestas de población haya estado encaminado antes del amplio empleo de la computadora, indudablemente el papel creciente de las encuestas aumentó debido a ella. Seguramente el aumento de uso de técnicas estadísticas multivariadas y de modelos causales se debe en parte a la disponibilidad de computadoras. Además, el desarrollo de modelos demográficos de simulación complejos que combinan características aleatorias individuales con aspectos estructurales del ciclo reproductivo o de hogares y familias, habrían sido imposibles sin la compu. tadora. Las simulaciones macrodemográficas se podrían haber hecho a mano, pero probablemente sin el detalle que ahora se incorpora, por ejemplo, en el modelo Bachue.

Ideas nuevas dentro del campo: demografía histórica, modelos biométricos, estimaciones indirectas, hogares y familias

La tercera fuente de cambios en demografía se refiere a las líneas de investigación nuevas cuyo origen está dentro del campo. Cuatro de estas líneas han surgido desde Roma; dos de ellas se derivan del trabajo de Louis Henry de los años cincuenta. La primera es el estudio de la demografía histórica a través de la explotación de los registros parroquiales. En este caso, el trabajo de Henry definió los problemas de investigación y previó la metodología de multiplicidad de estudios de aplicación preferencial en Francia, y también en diversas partes del mundo. Entre otros contribuyen tes pioneros se incluyen Goubert, Wrigley, Laslett, Dupaquier, Livi Bacci y Coale.

La segunda línea de investigación nueva y altamente productiva que se deriva del trabajo de Henry es el análisis biométrico de la fecundidad que fue iniciada independientemente por Dandekar. Este enfoque se ha fundido en otro, derivado del trabajo fundamental de Blake y Davis sobre las "variables intermedias" en el análisis de la fecundidad. El estudio de la fecundidad natural, la preocupación por la amenorrea durante el período de lactancia, los modelos matemáticos y estadísticos elaborados para ser aplicados al ciclo reproductivo, y la nueva 
"demografía de la anticoncepción", todos ellos integran esta línea que continúa enriqueciendo e informando el análisis de datos de encuestas; Leridon, Sheps, Srinivasan, D'Souza, Menken y Potter, entre otros, han con tribuido al refinamiento de los modelos originales. Gracias también a Bongaarts, el mismo enfoque básico ahora conduce a modeíos elegantes de descomposición simple de las diferencias de fecundidad entre poblaciones agregadas.

La tercera línea de investigación que surge dentro de la demografía, es el área de la estimación indirecta. El trabajo de Brass, iniciado durante los años cincuenta (Brass presentó un trabajo al respecto en la conferencia de Roma), concibe y ataca el problema de una manera novedosa. Este y otros enfoques relacionados se han aplicado con notable éxito, logrando asimismo medidas.más sólidas de la fecundidad y mortalidad en países en vías de desarrollo tanto como en los históricos, donde los niveles de enumeración son menores. El trabajo de Coale sobre cédulas modelo de fecundidad, nupcialidad y mortalidad y, junto con Demeny, sobre las poblaciones estables implicadas, ha sido central. Merecen ser destacadas las Naciones Unidas por la elaboración de las primeras tablas de vida modelo, al igual que Lederman por las primeras tablas más flexibles. Bourgeois-Pichat fue e] primero que aplicó los modelos de población estable a.la estimación de tasas vitales. Más recientemente, Somoza, Cho, Hil], Trussell, Zlotnick, y Rashad han avanzado en esta área. La colección completa de tablas modelo, cédulas y procedimientos y preguntas de encuesta que el demógrafo actual utiliza con naturalidad, creció durante los últimos treinta años.

La demografía de hogares y familias, íntimamente ligada a la nupcialidad y el divorcio, es la cuarta linea que ha experimentado un crecimiento rápido durante las décadas recientes. Los problemas definidos aquí son extensiones naturales de la demografía 'atómica' tradicional sobre individuos, y, aparentemente, los demógrafos fueron tan responsables de su inicio como los antropólogos e historiadores sociales. Aunque sea difícil ejemplificar un sólo trabajo seminal, indudablemente la colección compilada por Laslett y Wall se presenta como un paso importante. $\mathrm{El}$ análisis conductista de las causas y consecuencias de la composición de parentesco en los hogares todavía se encuentra en sus inicios, pero eventualmente deberán esclarecerse temas como la distribución del ingreso y la oferta de fuerza de trabajo, entre otros.

Se han mencionado cuatro áreas: demografía histórica, modelos de fecundidad biométricos y de variables intermedias, métodos de estimación indirecta, y la demografía de la familia y del hogar. Se observa inmediatamente, que estas líneas son fundamentalmente descriptivas; sólo de manera remota se ofrecen y someten a prueba algunas hipótesis sobre el comportamiento. Los métodos utilizados pueden ser más o menos teóricos, pero las teorías son de carácter matemático, estadístico o biológico, y poco se preocupan por las motivaciones. Sin duda esto explica el progreso rápido en estos campos, y la firmeza relativa de los hallazgos.

\section{Innovaciones de otros campos}

La cuarta fuente de cambios son las innovaciones introducidas desde otros campos, sobre todo de la estadística y la economía. 
De la estadística se ha recibido una variedad de modelos estocásticos de población, introducidos y aplicados por Pollard, Sykes, Le Bras y Hoem. Originalmente se esperaba que estos modelos ayudaran en las proyecciones, sobre todo proporcionando intervalos de confianza. Mientras, se estudia intensamente el empleo creciente de métodos estadísticos de series cronológicos, utilizados por Saboia y McDonald, que prometen tener mayor relevancia inmediata para las proyecciones, al menos en los países desarrollados; este enfoque requiere de más atención por parte de los demógrafos de lo que hasta ahora ha recibido. La estadística no ha penetrado aún en gran escala el área de las estimaciones indirectas; aparentemente porque las distorsiones en los datos no conducen al valor esperado de cero; de todos modos esta área se presta a una síntesis.

Además, la estadística ha proporcionado a la demografía una multiplicidad de modelos y procedimientos que ahora son comunes. Un breve listado incluye técnicas de ecuaciones simultáneas, procedimientos para el análisis de variables dependientes binarias, tales como probito, logito y el modelo de función del riesgo, también como el modelo loglineal para el análisis de interacciones en las tablas de contingencia. Estos y otros modelos permiten a los demógrafos lograr un mayor ajuste entre sus procedimientos estadísticos y las teorías a las cuales se aplican estos modelos.

Diversos aportes de la economía se patentizan en el período desde Roma, y se suceden a paso acelerado. Aunque en Roma hubo por lo menos cinco trabajos que trataron el crecimiento de la población, la formación de capital y el desarrollo en los países menos desarrollados, el primer intento sistemático y detallado de crear un modelo y simular las relaciones entre este conjunto de variables se debe a Coale y Hoover, pocos años más tarde. Tomando como ejemplo su libro sobre la India, han aparecido docenas de modelos más complejos y flexibles tanto para los países desarrollados como para los menos desarrollados.

Es posible que la contribución más dramática, más vigorosamente discutida, y más central de la economía haya sido la llamada "nueva economía del hogar", cuyos orígenes se deben a Becker, Mincer, Willis y trabajos relacionados de Leibenstein.

Este enfoque se ha aplicado a la fecundidad y a la oferta de fuerza de trabajo, pero en forma creciente también, a otros problemas. A pesar de las controversias suscitadas ha ejercido un impacto enorme sobre la demografía, y en ja actualidad continúa ejerciéndolo. En diversos sentidos parece ofrecer una formalización de antiguas ideas sobre los cambios y diferenciales en la fecundidad, toda vez que se logra superar las diferencias de lenguaje; asimismo, como otras teorias, ésta se basa en los efectos de la modernización y el desarrollo sobre los roles de la mujer y las inversiones en los hijos. En oposición a otras teorías, sin embargo, da poca importancia a los cambios en las preferencias.

Habría que mencionar otras tres contribuciones. La primera es el análisis descriptivo de Kuznets en cuanto a los aspectos estructurales del desarrollo económico-demográfico: este importante trabajo ha sido prácticamente ignorado por los demógrafos. Segundo, derivado de otra tradición kuznetsiana, y con huellas claramente maltusianas, se encuentra el trabajo de Easterlin sobre las fluctuaciones amplias de fecundidad en los países desarrollados. Este trabajo ha llamado mucho la atención, quizá en parte porque se lo puede considerar como 
una teoría demográfica sobre el cambio demográfico, a pesar de sus orígenes económicos. Tercero, La teoría general de población, de Sauvy, está llena de reflexiones ricas sobre la población y la macroeconomía, reflexiones que los demógrafos económicos yerran al no explotar.

Tal vez la mayor contribución de la economía, sin embargo, no ha sido la de proveer alguna teoría en particular, sino servir como ejemplo de una ciencia social del comportamiento, que, al igual que la demografía, cuenta con datos abundan. tes, y se ocupa de desarrollar modelos matemáticos formales y cerrados de procesos de formulación de decisiones o interacciones a nivel macro, y de estimaciones formales estadísticas y prueba de hipótesis. Este ejemplo ejerce una influencia importante sobre la demografía, con probabilidades de aumentar en el futuro.

UNA CARACTERIZACIÓN MÁS AMPLIA DE LAS ÁREAS DE CAMBIO Y DE PROGRESO

Hasta aquí se ha tratado de interpretar los cambios en la demografía utilizando un modelo simple que considera a la demografia como si fuera una economía en vías de desarrollo, persiguiendo una acumulación simple, creando tecnología nueva, prestando también tecnología nueva y aprovechando los flujos de capital extranjero. Debería existir acuerdo respecto de que el notable aumento de la población de demógrafos ha estimulado el crecimiento de conocimientos y de entendimiento en este campo, no obstan te haberse o no deprimido la tasa del producto per capita. Pero sería útil también considerar la posición del producto, y el grado en que responde a la composición de la demanda de nuestro producto. Aquí se encuentran varios ejes útiles para la discusión: micro-macro, conductista-descriptivo, causas-consecuencias.

\section{Micro-macro}

Se ha sugerido que los avances tecnológicos, sobre todo la computadora, han favorecido la recopilación y el análisis de datos a nivel individual (o. a nivel del hogar); esto, junto con los aportes teóricos relevantes provenientes de los campos de la economía y la sociología, y un interés por la heterogeneidad de variables intermedias de la fecundidad, han conducido a una preferencia pronunciada por el micro-análisis, al costo del análisis a nivel agregado. El abandono relativo de los trabajos de Kuznets y Sauvy es síntoma de esta tendencia.

\section{Conductista-descriptivo}

Otra manera de considerar el campo es ordenándolo según un eje "conductista"-"descriptivo". Se ha tratado de explicar la conducta y modelar las consecuencias de los cambios demográficos, en lugar de sujetarse a la demografía descriptiva. Digo esto, a pesar del hecho de que, según mi punto de vista, todas las innovaciones importantes dentro de la demografía hasta el presente, se com- 
prueba son principalmente descriptivas. El movimiento hacia el análisis de la conducta llega desde fuera de la demografía, y particularmente se debe a tradiciones y avances en la sociología y la economía.

\section{Causas-consecuencias}

La tercera distinción importante se establece entre el estudio de las casuas de los cambios demográficos y el de sus consecuencias. Las consecuencias demográficas de mayor interés actual no se perciben a nivel del individuo o de la familia, sino a nivel de la sociedad, mientras que para el comportamiento de la fecundidad la situación, en general, es inversa ya que las causas operan a nivel individual. Por lo tanto, es consistente con las observaciones anteriores ya que los estudios sobre las causas de los cambios demográficos y sobre todo los que tratan la fecundidad o migración, han aumentado a costa de los estudios sobre sus consecuencias. En la reunión de Roma, casi un cuarto de las sesiones se dedicaron exclusivamente a las consecuencias socioeconómicas de los cambios poblacionales. En Manila, según mis cálculos, Ja proporción fue quizá la mitad. En cambio, la proporción de sesiones dedicadas a la fecundidad aparentemente se duplicó.

¿Cómo se explica este cambio? Indudablemente no se debe a que la urgencia por una mayor comprensión de las consecuencias sea menor. Nuevamente, sospecho que el incremento de los avances de la tecnología ha privilegiado los microestudios, y que los estimulantes desarrollos teóricos y metodológicos que llegan desde fuera del campo también han privilegiado los estudios a nivel micro. Si en la actualidad quisieran estudiarse las consecuencias del crecimiento de la población a nivel macro, los tipos de datos que se utilizarían, las teorías y los métodos estadísticos, serían más o menos los mismos de que se disponía hace treinta años. El progreso en esta área requiere ideas o enfoques nuevos y creativos y éstos siempre escasean.

Indudablemente ha sido poco halagüeño para los investigadores, el hecho de que en tantos estudios empíricos transnacionales no se hayan detectado relaciones fuertes entre tasas de crecimiento de la población y tasas de crecimiento del ingreso per capita.

Nuestra intención no es sugerir que no se han logrado avances útiles en el estudio de las consecuencias. Muchos han sido los trabajos localizados en los modelos de simulación en gran escala, derivados del prototipo de Coale y Hoover: en primer lugar, surgieron importantes desarrollos de los modelos de crecimiento más neoclásicos, y luego se dirigió la atención a la desagregación sectorial. También. se ha avanzado en cuanto a las consecuencias económicas y sociales de los cambios en la composición por edades, es decir, pensiones, ahorros, la composición de la demanda y efectos sobre el mercado de trabajo: se ha trabajado sobre las consecuencias de la migración, y un número cada vez mayor de estudios relaciona la influencia del crecimiento de la población sobre la distribución del ingreso. También es cierto, sin embargo, que es mucho menor y de una manera diferente de lo que se hace en el caso de los estudios de fecundidad. 


\section{EL STATUS DE LAS PRINCIPALES TEORÍAS DE LA DEMOGRAFÍA}

Nuestro enfoque, hasta aquí, ha consistido en categorizar y descomponer los cambios en demografía; quizá sería útil acercarse ahora a un punto de vista más sintético: localizarse en el status de las dos principales teorías unificadoras conductistas dentro de la demografía: la teoría de la transición demográfica y la teoría maltusiana.

\section{La teoria de la transición demográfica}

En las líneas siguientes, cuando me refiero a la "teoría de transición", me refiero a la teoría tal como se la entendió en la época de la conferencia de Roma, y no en su ampliación y modificación posterior. La teoría de la transición consideró que la causa fundamental del descenso secular de la fecundidad se debió al desarrollo socioeconómico, y más específicamente, a la urbanización, la industrialización y la educación. Diversas fuentes de influencia fueron condensadas: el desarrollo requiere y premia las mayores inversiones en la educación de los niños, ya que éstas aumentan sus costos directos y disminuyen sus contribuciones económicas directas; el desarrollo creó nuevas oportunidades para la mujer, alterando asimismo su papel dentro de la sociedad; de hecho, en gran medida otras instituciones asumieron las funciones económicas y sociales de la familia; se reemplazó el espíritu de racionalidad individualista por una orientación hacia las metas sociales; y el desarrollo condujo a una reducción de la mortalidad, la cual creó presiones para que la fecundidad bajara.

Esta teoria fue considerada bastante vaga, con poco poder de predicción preciso, y sujeta a ciertas excepciones obvias, tales como el patrón de cambio en la fecundidad francesa. Después de tres décadas de investigación cada vez más intensiva, tha cambiado nuestra perspectiva sobre los determinantes de la fecundidad?

La teoría de la transición en la medida de su alcance, aparentemente sigue viva y sana; existe acuerdo total, sin embargo, en que la teoría sólo enfrenta una parte del problema. Una visión de la fecundidad más amplia, ha hecho su aparjción combinando diversas ideas de las líneas de investigación ya descritas.

La teoría actual, tal como la desarrollaron Henry, Coale, Tabbarah, Freedman, Easterlin y muchos otros, distingue entre los factores que afectan a la fecundidad a través del número de hijos deseados, de la fecundidad natural y del empleo de anticonceptivos. La teoría de la transición, tal como fue caracterizada en Roma, y hasta donde está vigente, sólo gira en torno al primero de estos factores; a saber, el número de hijos deseados. Por lo tanto, en el presente se halla limitada. Como teoría del número de hijos deseados, en cambio, incluye casi todos los elementos básicos de las formulaciones teóricas actuales; por ejemplo, la teoría de la nueva economía del hogar representa la formalización de las relaciones entre la inversión en los hijos y los costos de oportunidad de la mujer al dedicarse a los hijos, siendo éstos, temas centrales en la teoría de la transición. Aunque la teoría económica contribuye de manera significativa al ilustrar algunas implicaciones no percibidas en la formulación original, las reflexiones básicas son las 
mismas. Asimismo, la teoría de Caldwell, que se basa en revertir los flujos de riquezas, también aparece como una elaboración rica y detallada de las ideas fundamentadas por los teóricos de la transición.

Por otro lado, la teoría de la transición, como teoría del número de hijos deseados, no tomó en cuenta que -en ausencia de desarrollo socioeconómico, y como medida rápida para lograr un descenso en el número de hijos deseados- un gobierno podía intervenir de manera directa para modificar los incentivos y las normas; o sea, no se previó la posibilidad del éxito de China.

Entre las fuentes de mayor apoyo para la teoría de la transición como teoría de la demanda de hijos, se encuentran las encuestas actitudinales internacionales sobre el valor de los hijos, muchas de ellas organizadas por Caldwell y Pawcett. El análisis comparativo absorbente de Bulatao y otros muestra que según avanza la transición, se valoran cada vez menos los hijos como proveedores de seguridad en la vejez o por sus contribuciones productivas directas, y cada vez más por motivos de satisfacción psíquica; asimismo, los padres se sienten cada vez más atados por sus hijos. Mientras no sea esencial a la teoría, el comportamiento consistentemente caracteriza la intervención de estas varjables actitudinales e indudablemente se fortalece.

La teoría de la transición no ignoró por completo el aspecto de la oferta de fecundidad; sí estableció que la fertilidad no bajaba según avanzaba el desarrollo, y notó también que la baja de la mortalidad incrementó la oferta de hijos sobrevivientes. Ahora parece, sin embargo, que a veces la fecundidad natural aumenta con el desarrollo. Probablemente esto no se debe, como se pensó hace cinco años, a las mejoras en la nutrición, sino a la intervención de frenos culturales que afec$\tan$ a la fecundidad. Hoy en día en cualquier análisis cuidadoso de la fecundidad toma en cuenta estas influencias; de hecho, en algunos ocupa el primer plano, como se ve en los estudios recientes sobre África editados por Page y Lesthaeghe.

En cuanto a las técnicas para el control de la fecundidad, según la teoría de la transición, nunca se discutía la disponibilidad de métodos anticonceptivos suficientemente eficaces; por lo tanto, se anticipaba que el papel actual de lo que ahora se llaman "programas de planificación familiar", iba a ser bastante menor. Durante las últimas décadas muchos han abogado por este punto de vista, y esta situación todavía sigue vigente. Desde nuestro punto de vista, sin embargo, los acontecimientos y la investigación de los últimos quince anfos sugieren de manera convincente que los programas de planificación familiar pueden tener, y de hecho lo tienen, un impacto importante. Muchos estudios a nivel agregado, sean transnacionales o con respecto a varias áreas dentro de un solo país, demuestran el efecto del esfuerzo programático de la planificación familiar, independientemente de los indicadores de desarrollo; aunque también sea cierto que los programas son mucho más eficaces tratándose de áreas más desarrolladas; o sea, hay una relación fuerte interactiva.

Dada la complejidad de la imagen simplificada que ha sido trazada, no es sorprendente que sólo se hayan encontrado relaciones débiles con muchas excepciones entre el descenso de la fecundidad y el desarrollo. El estudio más ambicioso ha sido el Proyecto de Fecundidad Europea, en el cual participó un equipo internacional de investigadores bajo la dirección de Ansley Coale.

Este proyecto ha demostrado que sólo son posibles las generalizaciones más 
gruesas en cuanto a la relación entre el curso de la fecundidad real y el desarrollo. En cambio, la diferenciación marcada entre patrones de fecundidad según líneas culturales y lingüisticas provee una evidencia nueva y más fuerte sobre la existencia de algún tipo de proceso de difusión de normas, de la racionalidad empleada en la formación de la familia, del uso de anticonceptivos y de elementos desco. nocidos. Pero la importancia de tal difusión, hasta cierto punto independiente del desarrollo, sugiere una vez más que la intervención gubernamental tiene e] poder para jugar un papel independiente, y que los medios de comunicación masiva podrían apresurar el proceso.

Así es que las reflexiones e hipótesis en torno a la teoría de la transición encuentran el meollo en las teorías actuales de la demanda de hijos; hoy en día, sin embargo, se sitúan dentro de un marco más comprensivo. Retomaremos el tema de su status actual después de una breve discusión sobre Malthus.

\section{Teoria maltusiana}

La teoría maltusiana describe un sistema económico-demográfico en equilibrio con una retroalimentación negativa: el crecimiento de la población deprime el bienestar material, provocando que la fecundidad baje, la mortalidad suba y el crecimiento cese. Por lo tanto, no es únicamente una teoría de tasas vitales y bienestar económico, sino también una teoría sobre el tamaño de la población. Aunque algunos elementos individuales de esta teoría hayan recibido apoyo o crítica durante las últimas tres décadas, casi por completo se ha ignorado la teoría sintética del equilibrio. El "maltusianismo" de hoy suele limitarse a considerar los efectos negativos del crecimiento de la población; esta preocupación proporciona un punto de partida conveniente para comenzar la discusión.

Por el lado teórico, los que disienten con el punto de vista maltusiano han seguido por las bien elaboradas líneas de los críticos contemporáneos de Mal thus, y por la línea de los teóricos del estancamiento de los años treinta y cuarenta. Más recientemente, han surgido contribuciones importantes de varios estudiosos, y sobre todo de Boserup, quien ha brindado el relato más detallado y original de cómo el crecimiento de la población puede estimular el progreso, sobre todo dentro de las economías primitivas. Por el lado empírico, como ya se observó, muchos intentos de descubrir correlaciones entre las tasas de crecimiento de la población y las tasas de crecimiento del ingreso per capita solamente han encontrado coeficientes insignificantes y de signo inestable, siendo estos hallazgos sorprendentes para muchos.

Tenemos, por supuesto, los resultados de los modelos de simulación, pero éstos se basan en ecuaciones del comportamiento problemáticas. El informe del Club de Roma, y las alzas en los precios de los alimentos y de la energía, han revivido la preocupación por los límites del medio ambiente global; pero este fenómeno tiene poco que ver con la demografía como ciencia. Si existiera una generalizada creencia entre los demógrafos profesionales de que el crecimiento de la población es un problema humano muy serio, esa creencia tendría sus fuentes principalmente en otras disciplinas, en el sentido común, en la observación directa y en algunos cálculos obvios, en vez de algún hallazgo empírico 
profundo de la demografía o la economía. No se sugiere aquí que sea inapropiada la preocupación, sino que en tanto profesión ha sido sorprendentemente escasa la contribución efectuada aparte de los datos mismos y los cálculos $a$ priori basados sobre identidades y supuestos no verificados. Dos excepciones importantes son: los estudios transnacionales que demuestran un efecto de las tasas de crecimiento de la población sobre la distribución del ingreso, y el hecho de que aparentemente el tamaño relativo de los grupos de edad afecta de manera adversa el nivel de empleo o los ingresos en los países desarrollados.

Ahora confrontemos la teoría maltusiana con las causas del cambio poblacional, teoría que enfatizó el efecto positivo del ingreso relativo sobre la fecundidad es decir, relativo al estándar histórico, cultural o de clase social. Este propósito fue esencial a la teoría maltusiana de equilibrio, pero la mayor parte de los demógrafos modernos consideran que la relación tendría el signo opuesto, si es que existiera.

También ha recibido atención reciente el punto de vista maltusiano respecto a que la mortalidad dependía de manera significativa del ingreso o de la nutrición. Los historiadores médicos y económicos todavia debaten su relevancia para la Europa del siglo XIX. Durante la última mitad del siglo es claro que una gran parte de la disminución de la mortalidad se debe a las políticas deliberadas y no al desarrollo general o a la nutrición; Preston ha confirmado que en una muestra de países durante el siglo $\mathrm{xx}$, se debe al desarrollo la mitad del avance en la esperanza de vida al nacimiento.

Asimismo, múltiples investigaciones no han confirmado efectos negativos fuertes del crecimiento de la población sobre el bienestar, ni efectos positivos del bienestar sobre la fecundidad. No es de extrañar, pues, que casi nadie, salvo los antropólogos que estudian pequeñas poblaciones primitivas, contemple a la población como parte de un sistema de equilibrio automático. Dentro del contexto de la Europa preindustrial, Sauvy, Dupaquier, Schofield y Lesthaeghe han ofrecido interpretaciones homeostáticas interesantes. En cuanto al contexto moderno, sin embargo, la noción fundamental maltusiana del equilibrio requiere, para que sea relevante, de un grado de estabilidad estructural y tecnológica, y madurez económico-demográfica, que han estado ausentes desde la revolución industrial, aunque quizá estén aguardando fuera del escenario.

\section{Una interpretación}

En este apartado se ofrecerá una interpretación personal de las relaciones entre la teoría moderna de la fecundidad, la teoría de la transición demográfica, y la teoría maltusiana. La teoría de la transición consiste en un conjunto de aseveraciones respecto a cómo el desarrollo y la modernización afectan las opciones de fecundidad. Sin embargo, y hecho del cual son cada día más conscientes los demógrafos, el desarrollo y la modernización permean aspectos diferentes de la vida según pasos diferentes, afectando de manera desigual la aceptación de] uso de anticonceptivos, los comportamientos que afectan la fecundidad tales como la lactancia, y el número deseado de hijos. La teoría de la transición se preocupa sólo por el último, y por lo tanto es demasiado burda y restringida como teoría para ayudar a analizar Ja cronología real de los cambios 
en la fecundidad. Para esta tarea se adapta mejor la más detallada perspectiva moderna; al menos permite la interpretación y no la predicción. A través de un período largo, sin embargo, quizá uno o dos siglos, la permeación de la modernización seguramente se completará, y en este caso la fecundidad natural inicial se volverá irrelevante; se controlará mucho la fecundidad; y la teoría de la transición proporcionará, a grandes rasgos, una reflexión incisiva. Con esto no se quiere decir que la teoría moderna sea irrelevante a largo plazo, sino que el componente que se refleja sobre los cambios a largo plazo es esencialmente equivalente a la teoría de la transición anticuada.

A nuestro entender, sin embargo, estas teorías se muestran incompletas. No hay nada en ellas que explique por qué las poblaciones humanas agregadas por lo general tienen tasas de crecimiento próximas a cero, con tamaños de población cercanos a la capacidad en su medio ambiente, dadas las tecnologías disponibles.

Para el estado previo a la transición, se podría apelar como explicación al freno positivo. Pero los cambios de fecundidad durante y después de la transición no están, según la teoría, sujetos a una retroalimentación negativa, puesto que el maltusianismo considera poco importantes los efectos del ingreso. Además, los efectos asociados al precio del tiempo, a los flujos de riqueza, etc., tampoco se ven debilitados por el descenso de la población. En el presente se observan tasas de fecundidad, en el período posterior a la transición, sustancialmente por debajo del reemplazo en muchas partes del mundo industrializado; la mayor parte de los demógrafos opinan que habrá reducciones adicionales en el futuro. Nuestro punto de vista difiere: anticipamos que a largo plazo nuestras poblaciones llegarán otra vez, en promedio, a acercarse a una población casi estacionaria, con tasas de fecundidad globales cercanas a dos hijos por mujer.

Como consecuencia de esto sugerimos que la teoría maltusiana del equilibrio -reinterpretada adecuadamente- sí se aplicará a largo plazo, y que la teoría de la transición también se puede considerar como una teoría "transicional": describe el impacto de la modernización sobre sociedades con instituciones cuyas raices son tradicionales; no toma en cuenta el efecto retroalimentador de la reducción del crecimiento de la población sobre las instituciones sociales, económicas y políticas y sí sobre el contex to del proceso de decisiones individuales.

\section{Caminos Futuros}

Retomemos un tema ya discutido: ¿cuáles son los caminos nuevos más sugerentes en el campo? Por supuesto, los puntos de vista expuestos aqui son personales adémás de muy temues.

Hace mucho que la teoría de las poblaciones estables dejó de ser un área de crecimiento rápido. Tiene sus límites obvios, aunque maestros como Keyfitz o Bourgeois-Pichat rápidamente nos los hacen olvidar. Actualmente un área intensamente activa y estimulante es la de la creación de tasas vitales. Antícipamos, $\sin$ embargo, que a menos que se invente un enfoque verdaderamente nuevo, dentro de pocos años se habrán agotado las líneas principales y el ritmo de investigación decrecerá. Asimismo, los modelos de fecundidad biométricos han sido desarrollados ya en profundidad y son fructíferos y probablemente haya llegado el momento de la próxima etapa, en la que la tarea es anudarlos a modelos de comportamien- 
to que tratan de explicar sus niveles. Este es un enfoque muy sugerente, aunque queda la esperanza de que no excluirá la explicación de las variables intermedias en algunos contextos como estrategias alternativas e interrelaciones para lograr los resultados de fecundidad deseados, en vez de verlas como causas independientes.

La demografía de la senectud es un tópico de gran importancia para las políticas de población, pero hasta ahora, y con pocas excepciones, los demógrafos sólo han hecho contribuciones obvias. Esta afirmación también es aplicable a las implicaciones sociales y económicas del descenso del crecimiento de la población y del estudio de las consecuencias de la estructura y crecimien to de la población en general. Estos son tópicos que deberían estar maduros para desarrollarse, y serán retomados enseguida.

En oposición, muchas son las áreas que se encuentran en vías de cambio promisorio y aptas para un crecimiento rápido, tales como la demografía del hogar y de la familia, y sobre todo los determinantes y las consecuencias de la composición del hogar. También un área de investigación activa será el de los análisis de la salud y la mortalidad a nivel individual: basados en datos de nivel micro, existe un interés creciente por saber cómo las decisiones propias de los individuos afectan su salud y la de sus hijos. Seguramente la fecundidad seguirá recibiendo gran parte de la atención de los demógrafos, y esta área está en condiciones de aportar una síntesis basada en el trabajo de las últimas décadas, además de mostrar la apertura de nuevas sendas.

Un área problemática adicional es la interacción entre el desarrollo socioeconómico y la difusión de información, normas y patrones de comportamiento relacionados con el control de la fecundidad, y cómo las políticas de gobierno y las fronteras culturales les afectan. Existe la certidumbre creciente de que la difusión es de una importancia crítica, pero el progreso logrado en modelar este proceso es escaso. Tales investigaciones podrían contribuir a esclarecer la transición demográfica europea, además de dilucidar el papel de los programas de planificación familiar en el mundo contemporáneo.

Anteriormente se ha hecho hincapié en que los análisis de nivel micro han prosperado a costa del macro análisis. Sin embargo, en varias áreas se observan signos promisorios de una integración teórica y empírica de ambos. La discusión de Demeny de hace una docena de años sobre las externalidades, es un ejemplo, seguido ahora por McNicolls, y su preocupación por las influencias institucionales sobre los incentivos individuales referidos a la fecundidad; por Hermalin y Mason, que utilizando datos de la Encuesta Mundial de Fecundidad analizaron las influencias agregadas o comunitarias $y$, por la preocupación por las pensiones como externalidad positiva relacionada con la formación familiar. El análisis de estos temas debería dirigirnos hacia una visión más equilibrada de las causas y consecuencias demográficas en la década que sigue.

Como se observó antes, el estudio de las consecuencias de los cambios poblacionales sólo ha logrado un lento progreso. Esto también puede ser válicto para otras áreas para la década entrante. Sin embargo, se nota un èsfuerzo creciente por especificar las cuestiones importantes, y probablemente llevará a un progreso más satisfactorio. El estudio de las consecuencias de la migración en las áreas de expulsión y de recepción ha dado comienzo; asimismo, el tema relacionado de las consecuencias de la urbanización ya empieza a atraer una mayor atención. Otro 
tema sugerente es el de las consecuencias de la distribución por edades, sobre el cual ya aparece una literatura fascinante con contribuciones claves de Samuelson, Preston y Arthur.

La demografía histórica tiene por delante algunas décadas de trabajo relacionadas con la extensión de los métodos clásicos de reconstitución a nuevas poblaciones. Es dudoso que pueda profundizarse con este tipo de estudios. Existen abundantes y emocionantes cuestiones respecto a temas como la estructura de los hogares, las causas de las fluctuaciones de la mortalidad y las consecuencias de los cambios de tamaño de la población. Para encararlos se necesitan nuevas fuentes de datos y nuevos métodos. Algunos estudiosos en Francia y en Inglaterra están abriendo el camino.

\section{CONCLUSIONES.}

E] núcleo de la demografía siempre ha sido la descripción númerica esclarecida por el análisis de descomposición. Este núcleo sigue sano y en crecimiento, a través del desarrollo de nuevas técnicas y de nuevas descomposiciones, la definición de nuevas áreas sustantivas, los aportes de la computación, el uso de nuevas encuestas especiales, y otros caminos que aquí se trató de describir. Sin embargo, la influencia creciente de la economía y la sociología sobre la demografía no ha ayudado mucho a este núcleo descriptivo; más bien, y como efecto lógico, se ha dirigido hacia la explicación conductista de tendencias y diferenciales.

¿Qué es lo que se ha aprendido de esta área con un crecimiento tan rápido? Sería justo decir que existe poco de lo que denominamos "conocimientos sólidos". Existen ideas estimulantes, reflexiones, hipótesis; existen muchas teorías casi improbables, basadas en conceptos muchas veces imposibles de medir, $\mathrm{o}$, al menos hasta ahora, sin medir. Existen centenares de estudios empíricos contradictorios a nivel micro que explican proporciones infimas de la variación. Pero esto no: es de sorprender. Es "negocio común" dentro de las ciencias sociales, uno de los cuales, con certeza ha llegado a ser la demografía. ¿Es que sabemos por qué las parejas deciden tener un número diferente de hijos? Podemos citar docenas de influencias relevantes, pero hasta ahora ninguna de ellas permite pronosticar con confianza cuáles serán los futuros niveles de fecundidad, o explicar una porción grande de las diferencias en el comportamien to. ¿Cómo influye el crecimiento de la población sobre el desarrollo? Tenemos un grupo de estudios estadísticos inconclusos, un gran número de modelos de simulación basados en supuestos controvertidos a priori, y sólo alguna vez de acuerdo. La migración, como ya se observó presenta un cuadro más satisfactorio.

Por otro lado, si bien el núcleo mismo del conocimiento demográfico es descriptivo, no es menos cierto que el interés por el tema se deriva, en gran medida, de aquellas áreas de comportamiento demográfico que son los más oscuros, tales como las consecuencias del crecimiento. De ninguna manera se sugiere que deberíamos dirigir nuevamente nuestra atención a una demografía más descriptiva; todo lo contrario. Creemos estar bien encaminados en un esfuerzo por responder a preguntas más difíciles, las cuales inevitablemente tendrán respuestas menos sólidas y satisfactorias. Deberíamos apreciar, sin embargo, y seguir renovando nuestras raíces disciplinarias dentro de una tradición rica en la descripción numérica, la cual precisamente distingue a la demografía de muchas otras ciencias sociales. 